\title{
Selection for life span in Drosophila melanogaster
}

\author{
Leo S. Luckinbill and \\ Michael J. Clare
}

\author{
Department of Biological Sciences, Wayne State \\ University, Detroit, Michigan 48202.
}

Selection for reproduction at an early or a late age in life was applied to populations of $D$. melanogaster for 21 to 29 generations, with two experimental treatments of larval density. Populations with high and uncontrolled numbers of competing larvae responded strongly to selection for late-reproduction with the length of adult life increasing by as much as 50 per cent. In this treatment, selection produced true breeding long- and short-lived lines.

When populations of developing larvae were held low, however, longevity fluctuated wildly during selection, showing little overall response, as several previous tests of senescence theory have also found. These experiments suggest that life span is either physiologically limited in that environment, or populations are unable to respond because either phenotype/genotype correlations are reversed, or genetic variation is suppressed. The inability of former studies to obtain a response to selection appears to have resulted from the artifactual introduction of strong gene-environment interactions through the use of a competition-free environment.

\section{INTRODUCTION}

The early works of Haldane (1941), and Fisher (1930) first described the life history features of populations in terms of evolutionary theory, but it was not until Medawar (1952) and Williams (1957), that a specific explanation of the aging process incorporated both evolutionary and modern genetic concepts. Apart from the subsequent extension of these theories by Hamilton (1966), there has been little further revision of theory to date.

The experimental testing and verification of these theories has proceeded even more slowly. While early experimental studies by Clark and Maynard Smith (1955) found heterosis in the longevity of outcrossed populations, other studies by Comfort (1979) have described the patterns that emerge from the comparison of life span in different organisms. Collectively, the many such life historical and genetic studies do generally establish the existence of the genetic control of longevity, but they provide little more than implication toward the fundamental question of evolutionary interest "How do long and short life spans evolve?".

The longevity of individuals is thought to evolve in relation to demands by the environment for fitness at an early versus a late age in life.
Increased longevity is believed to evolve under circumstances where the reproduction of older individuals is favored. Delayed senescence, for example, should evolve when older individuals have an enhanced fitness because they can either produce greater numbers of progeny or provide an age-specific benefit to progeny. Also, repeated reproductions throughout a long life provide an effective counterbalance to a high juvenile mortality (Comfort, 1979; Schaffer, 1974; Murphy, 1968).

Alternatively, selection favoring reproduction early in life is thought to reduce life span indirectly. For example, in organisms that survive by colonising new or temporary environments, reproductive rates would be maximised by short developmental periods and heavy, early-reproduction. Because those species occupy temporary environments that are adequate for only a limited number of generations, a long life would be of little value and variants permitting early senescence would accumulate to eventually reduce life span.

Thus, a single central prediction issues from the evolutionary theory of senescence, independent of any mechanism of genetic control proposed. It is simply that selection favouring reproduction by long-lived individuals of advanced age should increase life span over that in which selection favours reproduction only by the young. 
Though the circumstances by which advantage is conferred on reproduction at old or young age may vary, those particulars are united in this single fundamental issue: selection for reproduction late in life should increase life span.

Despite the significance of this prediction, few experimental tests have been attempted. Moreover, those that have been performed have proven controversial in both results and conclusions drawn. Glass (1980) attempted an early test selecting for delayed senescence, but it was left to Wattiaux (1968) to perform the first relatively large scale study of this issue. In an unreplicated series of experiments, he compared the life span of populations of Drosophila subobscura reproduced at from 3 to 9 days or from 6 to 8 weeks of age. Selection began immediately after capture of the stocks from nature and continued for up to 11 generations in late-reproducing lines and 26 generations in earlyreproducing lines. Lack of methodological detail makes even a qualitative repetition of this experiment difficult but, the populations showed an increase in longevity in late-reproducing lines over those reproduced at an early age.

More recent studies have brought more careful experimental designs to bear on this issue. Lints and Hoste $(1974 ; 1977)$ performed a carefully controlled series of experiments with quite a different outcome. Here selection for early- or late-reproduction was applied by reproducing populations at either 4 days or 26 days after eclosion. The experimental populations used were hybrids descending from a four-way cross of four standard laboratory strains of Drosophila melanogaster, reproduced for 3-5 generations before selection. But more importantly, in order to prevent competition in the preimaginal environment from affecting adult life span, the density of developing larvae was held constant and low. Selection treatments were replicated and for the first time, longevity was measured at frequent intervals of one or two generations throughout the period of selection.

The enigmatic outcome of these studies is clearly inconsistent with not only the evolutionary theory of senescence, but also the notion that selection can alter life span at all. Longevity fluctuated wildly, dropping by about 70 per cent in both earlyand late-reproduced lines at the onset of selection and then abruptly recovered to original levels. Little net change resulted in 13 generations of selection, with remarkably wide fluctuations in the intervening generations.

This study was closely followed by another (Lints et al., 1979) in which selection for early- and late-reproduction was applied to a nonhybridised population of Oregon- $R$. Though the design of this experiment differed slightly, it was essentially a more careful version of the previous one, and the outcome was also consistent with the former study. Identical changes in longevity were noted for both the control and experimental lines with no difference between early- and late-reproducing lines.

In the absence of data to suggest otherwise, Lints and Hoste (1974) and Lints et al. (1979) conclude that longevity is not controlled by genes. Moreover, they advance the hypothesis that it is controlled by nongenetic maternal effects deriving from substances added to the egg at ovulation. The findings of these careful experiments together with this unique hypothesis, constitute a glaring contradiction for the evolutionary theory of senescence of the sort that can only be answered by the demonstration that life span does evolve under the influence of natural selection in a manner that is generally consistent with an evolutionary interpretation.

Subsequent experiments have attempted to supply such evidence. In Rose (1984) and Rose and Charlesworth $(1980 ; 1981)$, D. melanogaster was also subjected to schedules of early- and latereproduction. After twelve or thirteen generations of selection, late-reproducing lines had accrued a significant advantage in longevity. Analysis of trends also gave support to Williams' (1957) model of genetic control of aging, but their experiments differed from those of Lints and Hoste (1974) and Lints et al. (1979) in a number of ways. First the experimental populations were from a wild type population that had been carefully reared at high density to avoid inbreeding. Also populations had been under culture for enough generations to reach linkage equilibration before the start of selection. The density of developing larvae was uncontrolled in their experiments. And finally, unlike the repeated estimates in Lints' experiments, longevity and fecundity were measured only once, in the terminal generation.

Though Rose (1984) and Rose and Charlesworth's $(1980 ; 1981)$ results clearly support the convention that life span evolves, they are not exclusive of those of Lints and his coworkers. Because their studies measure life span only in the terminal generation of experiments, the possibility that large-scale erratic fluctuations may have occurred undetected in intervening generations cannot clearly be ruled out. Indeed, had Lints and Hoste measured life span only once, there are 
several points at which their populations would have appeared to fit a conventional evolutionary interpretation.

Thus, as it stands, two elegant and careful experiments validate the central assumption of all theory that life span evolves under selection, while two other equally powerful and well-designed studies contradict it. While the evolution of life span under the influence of natural selection cannot be doubted, its clear and unquestionable demonstration has yet to be performed for any species. Such a situation demands a critical retesting of theory with a re-examination of the methods and design of the foregoing experiments.

An independent test of theory not only promises the resolution of this dilemma, but also may uncover new empirical information. For, if artifactual effects are responsible for their results, Lints and Hoste (1974) and Lints et al. (1979) have, at the very least, raised important evidence of circumstances in which selection on life span is ineffective. Therefore, this study also attempts, as those before, to determine whether age-specific selection for reproduction modifies life span.

\section{MATERIALS AND METHODS}

A population of approximately 50 wild type $D$. melanogaster was obtained in four collections from a Michigan orchard and used to establish stocks for these experiments. Stocks were maintained separately for three or four generations at a density of more than 50 pairs per pint bottle and renewed at 10-14 days. To promote as extensive a variability as possible and following the methods of Lints and his coworkers $(1974 ; 1979)$, stocks were crossed such that each contributed equally in the creation of a four-way hybrid population. To permit the expression and equilibration of recombinants before selection, replicate lines of the hybrid population were then reproduced at 7-10 days for eight consecutive generations before selection was initiated.

Yeast-sucrose-agar medium with 0.01 per cent propionic acid added was used for both the maintenance of populations and experiments. Medium components were autoclaved separately and mixed before dispensing. To promote the growth of yeast in bottles, $0.5 \mathrm{ml}$ of an active suspension was introduced to culture bottles and allowed to dry for at least 48 hours before their use. It consisted of $6 \mathrm{gm}$ active dry yeast and $2.0 \mathrm{ml}$ of a 1.0 per cent solution of acetic acid diluted in $30 \mathrm{ml}$ of water. Population bottles were renewed at 48 hour intervals during experiments.

Longevity was estimated for 30 pairs of flies, isolated at one pair per shell vial. Active yeast was also added to vials 48 hours prior to use. In addition, the medium of these vials contained $0.04 \mathrm{gm}$ of Kodak (\#211) decolorizing carbon to provide a contrasting background for counts of eggs laid. Vials were renewed daily during measurement of longevity. All cultures were maintained at $25^{\circ} \mathrm{C}$ and at a 12:12 light/dark cycle. Adult longevity is measured here from the date of completed eclosion to the death of the individual. Total longevity includes the developmental period.

Larvae developed under two treatments. In one, the numbers of developing larvae were controlled and held at a low density as in Lints and Hoste (1974) and Lints et al. (1979). For this treatment, eggs were collected from replicate experimental lines on small egg-laying plates for up to 24 hours. These contained up to $6 \mathrm{ml}$ of yeasted medium and were inverted over the mouth of a standard pint bottle. After collection, the eggs were distributed at a density of 10 per vial to standard shell vials containing $15 \mathrm{ml}$ of medium, where development took place. This would equate to a density of approximately 40-50 larvae per pint bottle. The first 300 or so pupae to develop were sexed before eclosion and randomly reconstituted into populations of 50 pair per bottle for selection.

In the other treatment, the numbers of developing larvae were uncontrolled as in Rose (1984) and Rose and Charlesworth $(1980 ; 1981)$. Development in these lines took place at a much greater density than in controlled lines. Eggs were collected here over a period of 48 hours by simply retaining the population transfer bottles. Estimates indicate that densities ranged from between one and two orders of magnitude greater than that in density-controlled lines, with more than 1000 eggs laid per bottle. Here also, 300 or more pupae were removed and sexed before eclosion and adults were recombined into selection populations, as in other lines. Development time is measured for all treatments from the midpoint of the egglaying period to the midpoint of eclosion.

Selection for life span was applied to adults, as shown in fig. 1, by setting reproduction to occur at specific adult ages each generation. Mating and egglaying were continuous throughout life, but progeny were retained from early or late parental ages only. Progeny from days 2-6 after eclosion were retained in the early-reproducing lines. The age of late-reproduction was initially set at days 


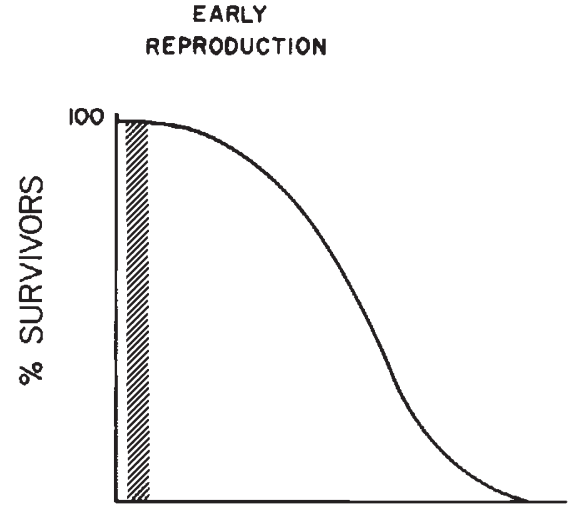

AGE

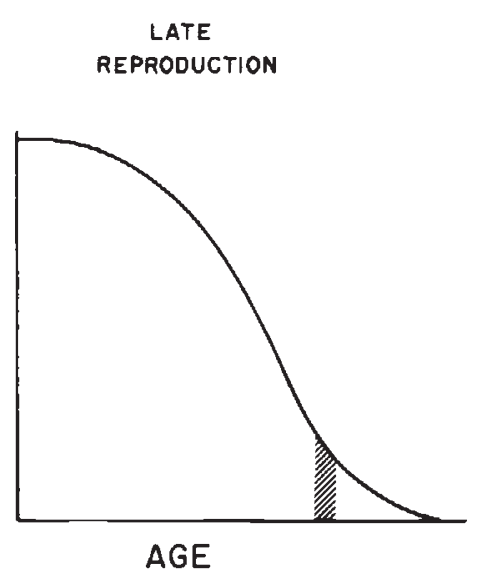

Figure 1 Selection for short life by early reproduction occurs at from 2 to 6 days after eclosion. Late-reproduction occurs only in individuals of greater then average life span, and should promote increased longevity.

22-26 after eclosion, but was increased, as lines responded to selection, to insure a continuous selective pressure. Late-lines always reproduced at an age greater than the mean life span for the population. By the 21 st generation, lines with uncontrolled larval-density were being reproduced after more than 70 days of adult life, while those with a constant larval-density were reproduced at about 55 days of adult life.

Three replicate early- and late-reproducing lines were each maintained under the controlled larval-density treatment. Two replicate early- and late-lines had uncontrolled larval-densities. Longevity was measured every fourth generation, except in the terminal generation which was measured after five generations.

For all selection lines of both treatments, an additional replicate population was also maintained. These sister lines were drawn from selection lines at each reproduction, and thus were treated identically to those used for the estimation of longevity. These populations were used to provide replacements for escaping or infertile individuals during longevity measurements and also to maintain selection lines at a constant population density of 50 pairs/bottle.

To determine the role of inbreeding in the changes observed in life span, replicates from latereproducing lines of the controlled-density treatment were outcrossed to one another. For this, additional females were collected from selection lines at reproduction and mass outcrossed to males of other replicate lines. Outcrossed lines were measured only once.

Near the termination of these experiments, selection for late-reproduction was reversed in treatments without density-control of larvae. After 20 previous generations of late-reproduction, these lines were subjected to five generations of earlyreproduction before measurement of their longevity.

\section{RESULTS}

The influence of the population density at which development takes place on the length of adult life has been documented in early studies by Miller and Thomas (1958), and more recently by Lints and Lints $(1969 ; 1971)$. Lints and Hoste (1974) designed their experiment to account for such an effect by holding the density of larvae controlled and low in populations under selection for adult life span. While their design is an admirable step toward a controlled experimental analysis of longevity, the non-stressful, non-competitive situation it provides may lack environmental components essential to the expression of the very genes of interest. The stresses and environmental circumstances which developing larvae characteristically undergo, constitute a significant adaptive force in the life history of the organism, by supplying critical environmental cues for the expression and/or repression of important genes. Our experiments account for the effects of those adaptive features by providing an environment with a significant level of competition.

Fig. 2 compares the results of 21 generations of selection for improved life span by reproduction at a late age, with 26-29 generations of selection for reproduction at an early age, in both experimental treatments. Tables 1 and 2 show the component measurements of adult life span and 


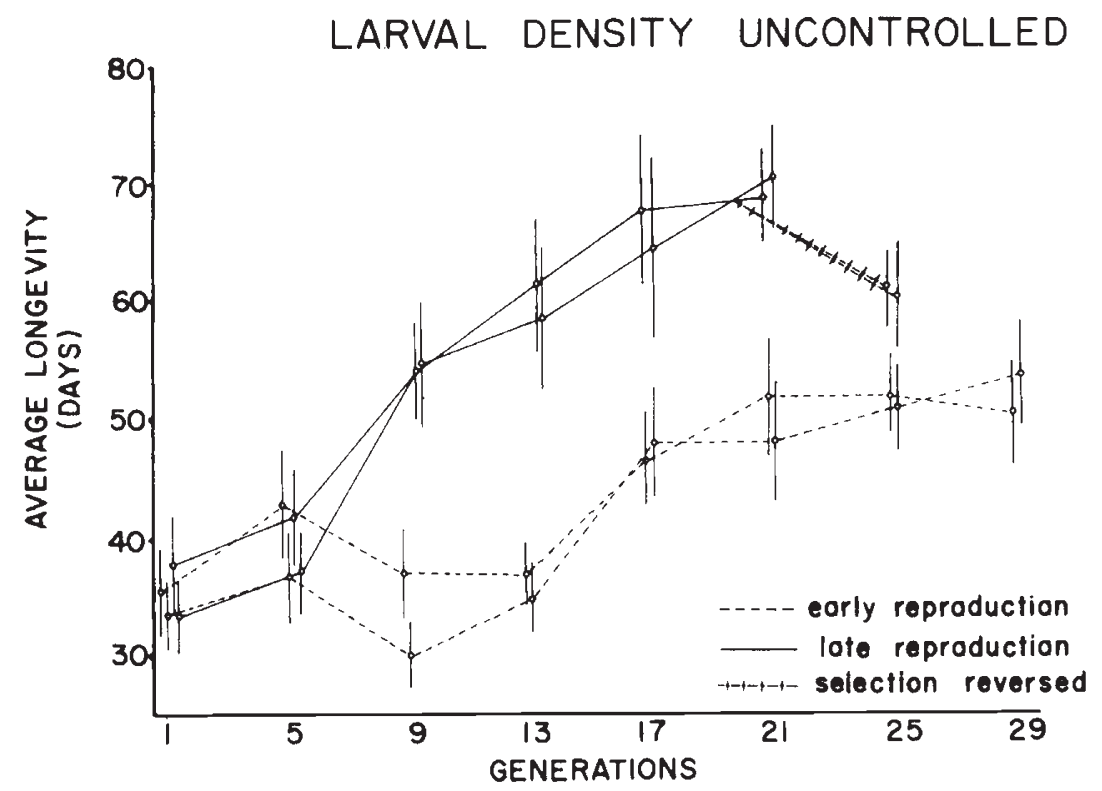

LARVAL DENSITY CONTROLLED

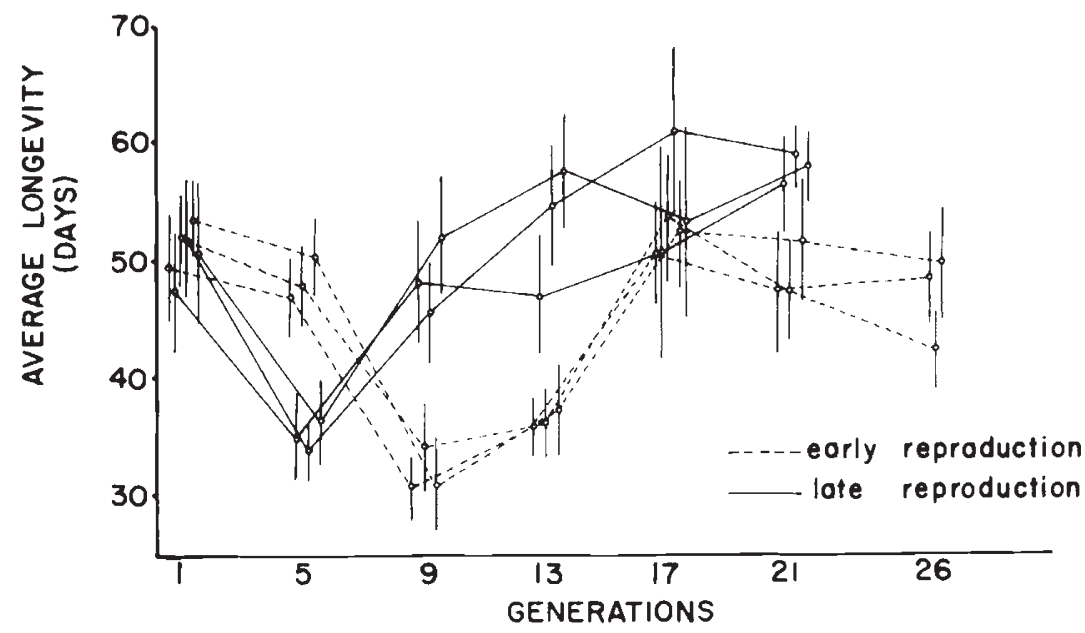

Figure 2 Selection for early- and late-reproduction under two experimental treatments is shown. The upper figure shows selection when the density of maturing larvae is uncontrolled, while selection at a controlled and low larval-density is shown below. The effect of five generations of reversed selection for long life are also shown (upper figure).

development for the experimental treatments of fig. 2 .

The control or noncontrol of the density at which larvae develop, gives distinctly different outcomes in these experiments. In populations with the numbers of maturing larvae uncontrolled, selection for late-reproduction produces a steady increase in life span from an average of 35 days at the start of selection, to more than 70 days by the terminal generation. Early-reproducing lines are low initially and then increase to an average of about 50 days by the 17 th generation, where they approximately remain until selection is terminated. Table 1 shows that adult life is from 47 to 56 per cent greater in late-reproduced lines than in early-reproduced lines. Thus, though increases occur in both early- and late-reproducing lines, continuous reproduction late in life strongly enhances longevity significantly over that in lines reproduced early. 
Table 1 Means and standard deviations of female longevity (in days) are compared in lines selected for early-and late-reproduction with larvae developing at uncontrolled densities. Development time is also shown

\begin{tabular}{|c|c|c|c|c|c|c|c|c|c|}
\hline & Generation & 1 & 5 & 9 & 13 & 17 & 21 & 25 & 29 \\
\hline \multirow{5}{*}{ Early reproduction } & Mean adult longevity & $19 \cdot 4$ & $24 \cdot 8$ & $26 \cdot 9$ & $23 \cdot 7$ & $33 \cdot 5$ & $40 \cdot 6$ & $38 \cdot 9$ & $37 \cdot 2$ \\
\hline & $\mathrm{S}$ & $9 \cdot 8$ & $12 \cdot 5$ & $10 \cdot 0$ & $7 \cdot 2$ & $11 \cdot 1$ & $13 \cdot 8$ & $9 \cdot 3$ & $12 \cdot 1$ \\
\hline & Mean adult longevity & $17 \cdot 4$ & $18 \cdot 6$ & $19 \cdot 9$ & $21 \cdot 6$ & $34 \cdot 9$ & $37 \cdot 0$ & $37 \cdot 8$ & $40 \cdot 8$ \\
\hline & $\mathrm{S}$ & $7 \cdot 4$ & $10 \cdot 7$ & $7 \cdot 2$ & $8 \cdot 5$ & $12 \cdot 5$ & $13 \cdot 7$ & $10 \cdot 9$ & $11 \cdot 9$ \\
\hline & Development & 16 & 18 & 10 & 13 & 11 & 13 & 11 & 12 \\
\hline \multirow{5}{*}{ Late reproduction } & Mean adult longevity & $27 \cdot 7$ & $30 \cdot 6$ & $43 \cdot 0$ & $51 \cdot 2$ & $58 \cdot 8$ & $57 \cdot 9$ & & \\
\hline & $\mathrm{S}$ & $11 \cdot 3$ & $10 \cdot 7$ & $10 \cdot 9$ & $15 \cdot 0$ & $18 \cdot 2$ & $11 \cdot 2$ & & \\
\hline & Mean adult longevity & $23 \cdot 4$ & $26 \cdot 0$ & $43 \cdot 6$ & $48 \cdot 3$ & $54 \cdot 4$ & $59 \cdot 5$ & & \\
\hline & $\mathrm{S}$ & $9 \cdot 7$ & $9 \cdot 5$ & $14 \cdot 3$ & $16 \cdot 3$ & $20 \cdot 6$ & $12 \cdot 3$ & & \\
\hline & Development & 10 & 11 & 11 & 10 & 9 & 11 & & \\
\hline
\end{tabular}

Table 2 Means and standard deviations for female longevity of lines selected for early-and late-reproduction with the density of larvae controlled are shown. Development time is also shown (in days)

\begin{tabular}{|c|c|c|c|c|c|c|c|c|}
\hline & Generation & 1 & 5 & 9 & 13 & 17 & 21 & 26 \\
\hline \multirow{5}{*}{ Early reproduction } & Mean adult longevity & $41 \cdot 4$ & $37 \cdot 8$ & $22 \cdot 6$ & $28 \cdot 9$ & $41 \cdot 3$ & $39 \cdot 1$ & $39 \cdot 4$ \\
\hline & $\mathrm{S}$ & $12 \cdot 1$ & $8 \cdot 7$ & $7 \cdot 3$ & $6 \cdot 7$ & $11 \cdot 9$ & $13 \cdot 7$ & $10 \cdot 3$ \\
\hline & $\mathrm{S}$ & $10 \cdot 3$ & $9 \cdot 1$ & $9 \cdot 8$ & $8 \cdot 0$ & $14 \cdot 5$ & $11 \cdot 3$ & $8 \cdot 7$ \\
\hline & Mean adult longevity & $45 \cdot 5$ & $41 \cdot 2$ & $22 \cdot 7$ & $30 \cdot 1$ & $43 \cdot 1$ & $43 \cdot 4$ & $40 \cdot 5$ \\
\hline & S & 8.6 & 8.9 & $11 \cdot 3$ & $10 \cdot 2$ & $12 \cdot 1$ & $14 \cdot 0$ & $12 \cdot 7$ \\
\hline \multirow{6}{*}{ Late reproduction } & Mean adult longevity & $39 \cdot 2$ & $27 \cdot 9$ & $38 \cdot 9$ & $36 \cdot 8$ & $42 \cdot 5$ & $47 \cdot 3$ & \\
\hline & $\mathrm{S}$ & $13 \cdot 5$ & $10 \cdot 2$ & $14 \cdot 1$ & $14 \cdot 0$ & $24 \cdot 1$ & $10 \cdot 7$ & \\
\hline & Mean adult longevity & $43 \cdot 5$ & $26 \cdot 6$ & $36 \cdot 5$ & $44 \cdot 5$ & $52 \cdot 8$ & $49 \cdot 6$ & \\
\hline & $\mathrm{S}$ & $14 \cdot 3$ & $6 \cdot 8$ & $11 \cdot 5$ & $13 \cdot 6$ & $19 \cdot 4$ & $7 \cdot 8$ & \\
\hline & Mean adult longevity & $42 \cdot 5$ & $29 \cdot 2$ & $43 \cdot 0$ & $47 \cdot 3$ & $45 \cdot 0$ & $48 \cdot 8$ & \\
\hline & $\mathrm{S}$ & $16 \cdot 0$ & $10 \cdot 0$ & $13 \cdot 3$ & $12 \cdot 9$ & $21 \cdot 7$ & $8 \cdot 1$ & \\
\hline
\end{tabular}

Between generations 17 and 21 , longevity continues to increase in one line, but does so less strongly in the other replicate. Selection studies often include a relaxed selection line at this point to show the effect of removing the type of selection applied. Since selection here is for age-specific reproduction, a relaxed selection treatment would have to include the simultaneous contribution of all age classes at reproduction. Relaxed selection such as this was not applied here, but it is of interest also to determine the extent of variation that remains for life span. To this end, selection was reversed at generation 20 to favour early-reproduction. Fig. 2 shows that five generations of reversed selection effectively reduces longevity by about seven days. Variability in the genetic elements controlling life span is apparently not exhausted by the 21 st generation of selection.

Selection in treatments with the numbers of larvae controlled produces a distinctly different overall outcome from that of lines without density control. Freedom from larval competition in the eight generations preceding selection is manifested by a more rapid development and also by a much higher average longevity at the onset of selection than in treatments with larval-density uncontrolled. The life span of late-lines drops from an average of about 50 days to about 35 days before returning to its initial level, from where it gains only about 7 days over the next 12 generations. Early-lines also decline at the start of selection and then rebound in a delayed fashion to their original values, where they also remain. Twenty-one generations of selection, for late-reproduction fails to produce a substantial increase in longevity.

Early-lines of both treatments show similar declines, beginning most strongly at the fifth generation. Although the possibility that this may have resulted from the same experimental disturbance cannot be completely ruled out, such an explanation seems unlikely. By the fifth generation, earlyreproducing lines of the two treatments were no longer synchronous, and differed in their starting date by more than 2 weeks. Additionally, late-lines in the same environment and media show no corresponding trend. 
During selection in the controlled-density treatment, a check was performed to determine whether fluctuations of life span in that treatment could possibly be the result of in vitro inbreeding. In that, mating and the relative contribution of individual females to the next generation are uncontrolled in these experiments, inbreeding could conceivably result from any factor causing the over-representation of small numbers of females at reproduction. Inbreeding followed by selection against inbreeding could produce the pattern observed by Lints and Hoste (1974), and in this study. To determine this, replicate late-selected lines of larval-density controlled treatment were outcrossed to one another at the ninth generation of selection. At this point, the lines were in the midst of the widest fluctuation in longevity that they undergo and therefore were presumably not fixed with respect to any particular genotype.

Table 3 compares the longevity of females in replicate lines of the controlled-density treatment, to that of females from outcrosses between replicate selection lines. Nonhomosedascity of variances suggested the use of a Mann-Whitney UTest, although the outcome is identical for a T-test.

Table 3 Female longevity of density-controlled selection lines is compared for inbred and outbred replicate lines

\begin{tabular}{llll}
\hline Selection line & Mean longevity & $\mathrm{S}$ & $\mathrm{N}$ \\
\hline $\mathrm{L}_{1}{ }^{*}$ & $49 \cdot 9$ & $14 \cdot 1$ & 30 \\
$\mathrm{OL}_{1}{ }^{*}$ & $51 \cdot 1$ & $12 \cdot 1$ & 30 \\
$\mathrm{~L}_{2} \dagger$ & $47 \cdot 5$ & $11 \cdot 5$ & 30 \\
$\mathrm{LL}_{2} \mathrm{~L}_{3}$ & $52 \cdot 6$ & $11 \cdot 1$ & 30 \\
$\mathrm{OL}_{3} \ddagger$ & $54 \cdot 0$ & $13 \cdot 3$ & 30 \\
\hline
\end{tabular}

Comparison of outcrossed lines with controls by MannWhitney U test

\begin{tabular}{llll} 
Lines & $\mathrm{N}$ & $\mathrm{U}$ & $\begin{array}{l}\text { Attained } \\
\text { significance }\end{array}$ \\
\hline $\mathrm{L}_{1} / \mathrm{OL}_{1}$ & $30 / 30$ & $475 \cdot 5$ & $p>0 \cdot 35$ \\
$\mathrm{~L}_{2} / \mathrm{OL}_{2}$ & $30 / 30$ & $554 \cdot 0$ & $p>0.06$ \\
$\mathrm{~L}_{3} / \mathrm{OL}_{3}$ & $30 / 30$ & $459 \cdot 0$ & $p>0.44$
\end{tabular}

* Outcrossed to $\mathrm{L}_{2}$ males.

$\dagger$ Outcrossed to $\mathrm{L}_{3}$ males.

$\ddagger$ Outcrossed to $\mathrm{L}_{1}$ males.

Outcrossing increased life span by up to 5 days in one line, but no outcross significantly increased longevity. This outcome is surprising, not only in itself, but also because the population was at that time undergoing a large fluctuation in longevity, presumably in response to changing gene frequencies. If the lack of response of these populations to outcrossing does not suggest isogeny in the lines, then it shows the existence of other powerful controls sufficient to suppress the effect of outcrossing. Certainly the variation in longevity in replicate density-controlled lines does not appear to result from inbreeding depression.

Of interest here also is the fact that the fluctuations in longevity of these lines are qualitatively identical to those found by Lints and Hoste (1974)! Despite the differences in the origin and treatment of stocks selected, the same pattern in fluctuations of early- and late-lines occurs in this study. These experiments, however, offer the unique comparative advantage of applying two treatments simultaneously to identical original populations. Therefore, since lines with uncontrolled-density respond to selection, the nonresponse of controlled-density lines here and in Lints' studies cannot be ascribed to either the 1) genetic constitution of the stocks used, 2) the amount of inbreeding to which stocks were subjected before selection, 3) applying selection to non-equilibrium populations, or 4) the hybridisation event that stocks underwent before selection. Treatments of this study differed only by the density at which larvae develop. The nonresponse of populations under the controlleddensity treatment can be attributed, therefore, only to the developmental environment.

The overall response of populations to selection in the two treatments is not a simple one. The longevity of early-lines in the uncontrolled-density treatment increases to about the same level as that maintained from the start of selection in the controlled-density lines. This suggests that there may be more than one factor operating during selection. A life span of approximately 50 days could conceivably constitute some sort of minimum threshold for longevity, set here by developmental and environmental circumstances, and beyond which the response to selection for late-reproduction is more direct.

Though the absolute behaviour of populations under both treatments of density is complex, the relative response of treatments under selection is clear. In the uncontrolled treatment changes in longevity are progressive and appear to result from genuine adaptive modifications. Fecundity varies in that treatment, however, and the effect of natural selection for improved fecundity should be considered in relation to the changes observed in longevity.

Lints and Lints $(1969 ; 1971)$ have shown that the longevity of $D$. melanogaster is extended by circumstances of intense larval competition. Meager resources can prevent larvae from easily 
reaching critical weights at various developmental stages and force a physiological extension of life on starving young and adults. Both longevity and developmental time increase with density of larvae under these circumstances, and adult fecundity is diminished. Can it be that an adaptation in the fecundity of late-reproducing, uncontrolleddensity lines occurs here and the consequent increase in the numbers of larvae has forced a physiological extension of longevity beyond that in early-lines?

The development time and comparative fecundities in early- and late-lines of the uncontrolleddensity treatment are inconsistent with that hypothesis. For, if the longevity of late-lines derived from extreme physiological stress of high larvel density, then development time would also be affected. But development shows no such effect in late-lines and in fact, is slightly less than that of the early-lines in the uncontrolled-density treatment. Furthermore, when early-lines reproduce (days 2-6), fecundity is up to fivefold higher than when late-lines reproduce. Therefore, extending longevity by density/stress should give early-lines the higher longevity instead of the opposite. Physiological prologation of life span through increased fecundity and developmental density may occur to some extent, therefore, but results indicate it is not the preponderant influence here.

Nevertheless, to test for possible effects on longevity of a variable density at development, fecundity was measured under selection conditions for noncontrolled-density populations in bottles. This was done by counting the eggs laid over 48 hours in bottles that had been cut, allowing the upper half to be removed. Developmental density was manipulated by removing or adding eggs to those laid by experimental populations, and the upper half replaced to allow pupation.

To determine whether life span can be made to vary this way, the density at development was altered over a fourfold range as follows; fecundity for early-reproducing lines was reduced by threefourths from that of a control population, while in late-reproducing populations, it was increased fourfold over that of the control. Longevity was measured as before. If the difference in life span of early-and late-reproducing populations here is purely physiological and is caused by an adaptive or artifactual effect of a varying density at development, then the longevity in populations with altered developmental density will diverge from that of control populations.

Table 4 shows that no such shift occurs, however, and varied developmental density, at least over this range, has little effect. Longevity in the uncontrolled-density treatment shows no particular dependency on changes in fecundity and appears to have responded to selection in a more or less conventional manner.

\section{CONCLUSIONS}

Our experiments have shown that:

(1) Twenty-one generations of selection for latereproduction dramatically increases longevity in lines with the density of larvae uncontrolled.

(2) The enhanced longevity of these lines does not result from the physiological prolongation of life that can accompany the starvation of larvae.

(3) Selection for longevity was ineffective in lines where the number of developing larvae was held low with wild fluctuations occurring, as in Lints and Hoste (1974). Precisely why selection fails and life span fluctuates is unclear, but the wide fluctuations observed appear to result from the variable expression of genes, rather than their replacement by selection.

These experiments independently confirm the results of Rose (1984) and Rose and Charlesworth $(1980 ; 1981)$. Selection increased longevity here

Table 4 Indicates the changes in average female longevity (in days) under selection conditions in botties, caused by variation in developmental density. Developmental-density was reduced for early-reproducing populations and increased for late-reproducing populations

\begin{tabular}{|c|c|c|c|c|c|}
\hline Selection applied & Treatment & Replicate & Development & Adult longevity & $\mathrm{S}$ \\
\hline \multirow[t]{4}{*}{ Early reproduction } & Control & 1 & 15 & $39 \cdot 6$ & $9 \cdot 6$ \\
\hline & & 2 & 13 & $39 \cdot 2$ & $16 \cdot 3$ \\
\hline & Density of eggs reduced by $3 / 4$ & 1 & 12 & $39 \cdot 9$ & $12 \cdot 0$ \\
\hline & $(350-400$ eggs $)$ & 2 & 12 & $41 \cdot 7$ & $9 \cdot 6$ \\
\hline \multirow[t]{4}{*}{ Late reproduction } & Control & 1 & 15 & $64 \cdot 5$ & $21 \cdot 0$ \\
\hline & & 2 & 14 & $63 \cdot 9$ & $15 \cdot 1$ \\
\hline & Density of eggs increased by $4 \times$ & 1 & 12 & $62 \cdot 7$ & $15 \cdot 0$ \\
\hline & $(1000-1200$ eggs $)$ & 2 & 12 & $66 \cdot 4$ & $14 \cdot 4$ \\
\hline
\end{tabular}


as in their study. Other more specific predictions of the Williams (1957) evolutionary model of senescence have been tested with these strains (Luckinbill et al., 1984), and they appear also to confirm that model. This study extends and completes those data. But of equal interest is the fact that these experiments also independently confirm the studies of Lints and Hoste (1974) and Lints et al. (1979). 21 generations of selection in the controlled-density treatment failed to produce more than a slight difference between early- and latereproducing lines. Apart from a possible relationship with fecundity which is unsupported by the results of the other treatment, the cause of such an outcome is unclear. It immediately poses the question "Why should conditions of the larval environment determine the efficacy of selection on adults?".

One possible, albeit unlikely, hypothesis is that the circumstance of development in vials (at least at low density) somehow exerts a direct and purely physiological effect on larvae limiting adult life span, while conditions in bottles do not. It is certainly not clear from the performance of populations in these experiments what such a factor might be or how it could operate to limit or alter phenotype/genotype correlations in characters under selection. Conditions in vials appeared to be much more nearly optimal than for competing populations in bottles, with viability in controlleddensity populations usually at more than 95 per cent.

Another hypothesis can also be advanced to explain these results, based on the ideas of Lerner (1954) and Waddington $(1957 ; 1961)$. Simply, it is that in the controlled-density treatment, a low and controlled number of larvae develop in a less stressful environment, allowing developmental buffering systems to suppress the expression of genetic variation in favour of a single phenotype. Selection is then prevented from operating effectively. Conversely, circumstances of high uncontrolled numbers of larvae are competitive, with stressful developmental conditions resulting. Developmental buffering systems would be ineffective there, permitting the expression of genetic variants and the operation of selection.

Evidence is abundant from Drosophila that the larval environment can determine the effectiveness of selection on adults. In earlier studies, Robertson $(1961 ; 1963 ; 1964 ; 1966)$ showed that in situations where selection altering body size is ineffective, placing $D$. melanogaster under even moderate nutritional stress by limiting RNA or protein will cause it to respond to selection. Resulting adapta- tions were not necessarily specific to the particular stress applied. And furthermore, whether the phenotype of crosses between selected lines and controls showed dominance by a single gene or additivity by several depended upon whether larvae developed in respectively optimal or stressful environments.

More recently, Parsons (1977), Derr (1980) and Murphy et al. (1983) have shown that the nature of variation in life history traits, including longevity, changes with the degree of environmental stress. Thus the type of environment can determine the nature of the genetic variation exposed to selection through gene-environment interactions. Suppression of the expression of genetic variants by a single dominant allele in a nonstressful environment could, at least temporarily, keep selection from being effective.

Some very clear predictions issue from this hypothesis. If stress in the larval environment determines a more conventional expression of genes for life span, for example additivity, then $F_{1}$ individuals will exhibit an intermediate phenotype between that of the long- and short-lived parental lines. But, if a gene-environment interaction does alter the expressed variability at a densitythreshold here, then in the nonstressful controlleddensity environment the same $F_{1}$ population should show only the short-lived phenotype of unselected individuals. That is, the same genotype should manifest highly different phenotypes when raised at different developmental densities.

Clare and Luckinbill (1985) have tested these predictions using the strains of this study. Their results show that in the stressful, uncontrolleddensity environment, the phenotype of $F_{1}$ individuals is additively distributed, and intermediate between long- and short-lived parental lines. But when $F_{1}$ populations or even long-lived parental lines are raised at controlled density, adults are only short-lived. Indeed, adult life span is strongly mediated by the density at which larvae develop.

The results of Lints and Hoste (1977) and Lints et al. (1979) are fully interpretable in the light of this, as well as the discrepancy between their studies and those of Rose (1984) and Rose and Charlesworth $(1980 ; 1981)$. In the latter works and this, the density of larvae were uncontrolled and the resultant competition may well have stressed the population during development, allowing the expression of the appropriate genes. In addition to the study of Lints and Hoste (1974), others have also failed when applying selection to populations with larval-density controlled. Lints and Gruwez 
(1972), selecting for rapid and slow development in D. melanogaster, found wild fluctuations in developmental speed to occur. Flanagan (1980) also had puzzling results applying selection for longevity to populations with controlled-densities. Our hypothesis suggests that all of those studies and indeed, our own controlled-density lines, would have obtained a positive outcome if only selection had been applied long enough to affect the derepression of the appropriate genes.

Though both the genetic mechanism and environmental circumstances selecting for an increased life span have been specified in theory, several reasonable attempts at selection have failed to find any support for the notion that life span will evolve. This study, and that of Clare and Luckinbill (1985) show that selection for reproduction late in life can increase longevity, as theory predicts, and also that powerful gene-environment interactions influence adult life span and consequently, the effectiveness of selection. The inability of preceding studies to obtain sensible results during selection appears to have resulted from the artifactual introduction of these gene-environment interactions by controlling the density of developing populations to low levels.

Acknowledgements We thank Michael R. Rose for his comments on a previous draft of this work. This research was supported by grant AGO1812 from the National Institutes of Health (U.S.A.) to L.S.L. and Robert Arking.

\section{REFERENCES}

CLARE, M. J. AND LUCKINBILL, L. S. 1985. The effects of gene-environment interaction on the expression of longevity. Heredity, 55, 19-29.

CLARK, J. M. AND MAYNARD SMITH, J. 1955. The genetics and cytology of Drosophila subobscura. XI. Hybrid vigour and longevity. Genet. Res. (Camb), 26, 1-10.

COMFORT, A. 1979. The Biology of Senescence. Third Edition. Churchill Livingstone, Edinburgh.

DERR, J. A. 1980 . The nature of variation in life history characters of Dysdercus bimaculatus (Heteroptera: Pyrrhocoridae), a colonizing species. Evolution, 34, 548-557.

FISHER, R. A. 1930. The Genetical Theory of Natural Selection. Clarendon Press, Oxford.

FLANAGAN, J. R. 1980. Detecting early-life components in the determination of the age of death. Mech. Aging Dev., 13, 41-62.

GLASS, B. 1960. The influence of immediate versus delayed mating on the life span of Drosophila. In B. Strehler (ed.), The Biology of Aging. Amer. Inst. Biol. Sci. Vol. 6., Publ. 6, 185-187.

haldane, J. B. S. 1941. New Paths in Genetics. Allen and Unwin, London.

HAMILTON, W. D. 1966. The moulding of senescence by natural selection. J. Theoret. Biol., 12, 12-45.

LERNER, 1. M. 1954. Genetic Homeostasis. Wiley. N.Y.
LINTS, F. A. AND I.INTS, C. V. 1969. Influence of preimaginal environment on fecundity and ageing in Drosophila melanogaster hybrids. I. Preimaginal population density. Exper. Gerontol., 4, 231-244.

LINTS, F. A. AND LINTS, C. V. 1971. Influence of preimaginal environment on fecundity and ageing in Drosophila melanogaster hybrids. 111. Developmental speed and life span. Exp. Gerontol., 6, 427-445.

LINTS, F. A. AND GRUwEZ, G. 1972. What determines the duration of development in Drosophila melanogaster? Mech. Aging Dev., 1, 285-297.

LiNTS, r. A. AND HOSTE, C. 1974. The Lansing Effect Revisited. I. Life span. Exp. Gerontol., 9, 51-69.

LINTS, F. A. AND HOSTE, C. 1977. The Lansing Effect revisited. II. Cumulative and spontaneously reversible effects on fecundity in Drosophila melanogaster. Evolution, 31, 387404.

LINTS, F. A., STOLI, J., GRUWEZ, G. AND LINTS, C. V. 1979. An attempt to select for increased longevity in Drosophila melanogaster. Gerontology, 25, 192-204.

LUCKINBILl, L. S., ARKING, R., CLARE, M. J., CIROCCO, W. AND BUCK, 5. 1984. Selection for delayed senescence in Drosophila melanogaster. Evolution, 38(6), 996-1003.

MEDAWAR, P. B. 1952. An Unsolved Problem in Biology. Lewis, London.

MILLER, R. S. AND THOMAS, J. L. 1958. The effects of larval crowding and body size on the longevity of adult Drosophila melanogaster. Ecology, 39, 118-125.

MURPHY, G. I. 1968. Pattern in life history and the environment. Amer. Naturalist, 102, 391-403.

MURPHY, P. A., GiESEI, J. T. AND MANLOVE, M. N. 1983. Temperature effects on life history variation in Drosophila simulans. Evolution, 37, 1181-1191.

PARSONS, P. A. 1977. Genotype-temperature interactions for longevity in Drosophila simulans. Exp. Gerontol., 12, 241 244.

ROBERTSON, F. W. 1961. The ecological genetics of growth in Drosophila. 4. The influence of larval nutrition on the manifestation of dominance. Genet. Res. (Camb), 2, 346360.

ROBERTSON, F. W. 1963. The ecological genetics of growth in Drosophila. 6. The genetic correlation between the duration of the larval period and body size in relation to larval diet. Genet. Res. (Camb), 4, 74-92.

ROBERTSON, F. W. 1964. The ecological genetics of growth in Drosophila. 7. The role of canalization in the stability of growth relations. Genet. Res. (Camb), 5, 107-126.

ROBERTSON, F. W. 1966. The ecological genetics of growth in Drosophila. 8. Adaptation to a new diet. Genet. Res. (Camb), 8, 165-175.

ROSE, M. R. 1984. Laboratory evolution of postponed senescence in Drosophila melanogaster. Evolution, 38(5), 10041009.

ROSE, M. R. ANI) CHARLESWORTH, B. 1980. A test of evolutionary theories of senescence. Nature, 287, 141-142.

ROSE, M. R. AND CHARLESWORTH, B. 1981. Genetics of life history in Drosophila melanogaster. II. Exploratory selection experiments. Genetics, 97, 187-196.

SCHAFFF:R, W. M. 1974. Selection for optimal life histories: The effects of age structure. Ecology, 55, 291-303.

waddington. C. H. 1957. The Strategy of the Genes. Allen and Unwin, London.

WADDINGTON, C. H. 1961. Genetic assimilation. Adv. Gen., 10. 257-293.

WATtiauX, J. M. 1968. Cumulative parental age effects in Drosophila subobscura. Evolution, 22, 406-421.

Williams, G. c. 1957. Pleiotropy, natural selection and the evolution of senescence. Evolution, 11, 398-411. 